\title{
Infant Feeding and the Developmental Origins of Chronic Disease in the CHILD Cohort: Role of Human Milk Bioactives and Gut Microbiota
}

\author{
Meghan B. Azad ${ }^{1,2}$
}

Keywords: human milk, gut microbiota, chronic disease, breastfeeding, gut microbiome, CHILD Study

$\mathbf{T}$ HE INCREASING PREVALENCE OF CHRONIC DISEASES, sUch as allergies, asthma, and obesity, may be linked to early life exposures and experiences during critical periods of prenatal and early postnatal development. The CHILD study is a prospective longitudinal cohort study following nearly 3,500 families across Canada from pregnancy through early childhood. ${ }^{1}$ Data from the study are being used to explore how genes and the environment influence child health and development. One area of focus is the role of breastfeeding on the infant gut microbiome and long-term health outcomes.

A recent analysis of 2,553 infants from the CHILD cohort reported a dose-dependent inverse association between breastfeeding and body mass index (BMI) at 1 year of age (Fig. 1). ${ }^{2}$ Supplementation with formula by 6 months diminished the effects of breastfeeding on BMI, whereas sup- plementation with solid foods around this time had no significant effect. The study also found that infants who received pumped breast milk from a bottle in the first 3 months of life had a higher BMI at 1 year versus infants who were exclusively fed at the breast. Although it is not clear why the mode of breast milk delivery makes a difference, possible reasons include increased skin-to-skin contact and motherinfant bonding with breastfeeding, better self-regulation of milk intake at the breast, and potential loss of important bioactive components during freezing or refrigeration of pumped milk. A similar dose-response relationship was seen with breastfeeding and wheezing in the CHILD cohort. Among infants born to mothers with asthma, exclusive breastfeeding for 6 months was associated with a $62 \%$ reduced risk of wheezing within the first year; partial

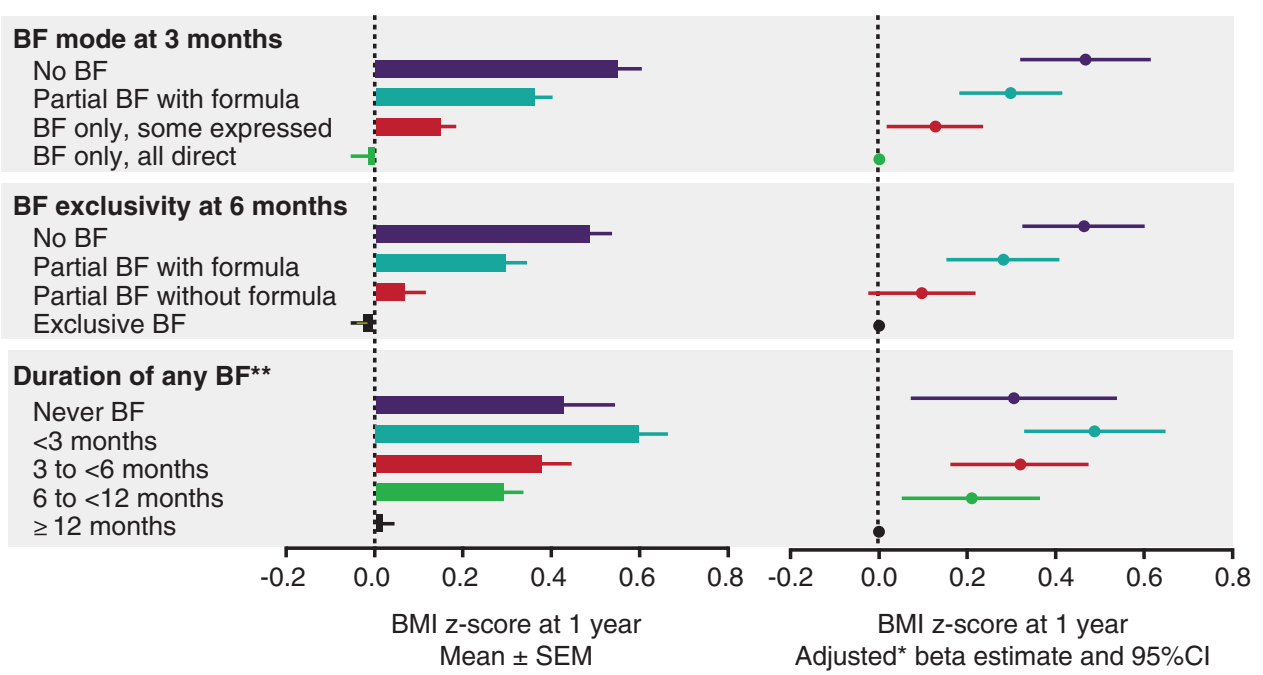

FIG. 1. Breastfeeding (BF) was inversely associated with infant BMI in the CHILD cohort. ${ }^{2} *$ Adjusted for maternal age, prepregnancy BMI, ethnicity, education, smoking, mode of delivery, parity; infant sex, and gestational age. **Mutually adjusted for each other. BMI z-scores are calculated according to the World Health Organization reference standard. BMI, body mass index. Reproduced with permission from Pediatrics, Vol. 142, e20181092, Copyright (C) 2018 by the AAP.

\footnotetext{
${ }^{1}$ Children's Hospital Research Institute of Manitoba, Department of Pediatrics and Child Health, University of Manitoba, Winnipeg, Canada.

${ }^{2}$ Manitoba Developmental Origins of Chronic Diseases in Children Network (DEVOTION), Winnipeg, Canada.

(C) Meghan B. Azad, 2019; Published by Mary Ann Liebert, Inc. This Open Access article is distributed under the terms of the Creative Commons License (http://creativecommons.org/licenses/by/4.0), which permits unrestricted use, distribution, and reproduction in any medium, provided the original work is properly cited.
} 


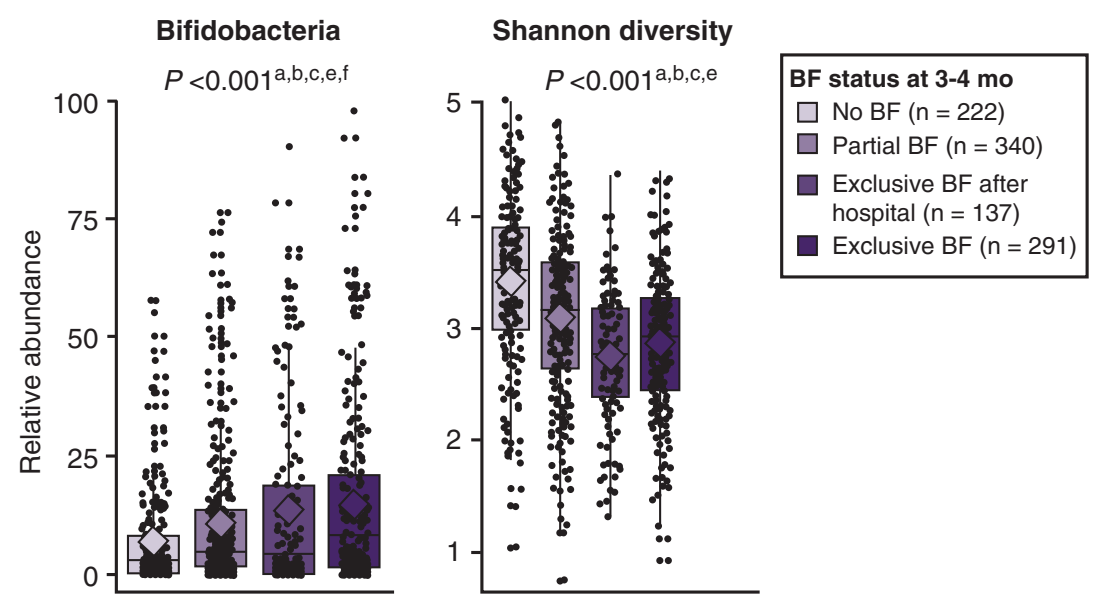

breastfeeding (without formula) was associated with a $37 \%$ reduced risk. ${ }^{3}$ A separate study found that, compared with direct breastfeeding, any other mode of infant feeding (i.e., pumped milk and formula) at 3 months was associated with an increased risk of possible or probable asthma by 3 years of age. ${ }^{4}$

The impact of breastfeeding on obesity and asthma may be related to its effects on the infant gut microbiome. In the CHILD cohort, breastfeeding was associated with lower richness and diversity of gut microbiota at 3 to 4 months of age (Fig. 2). ${ }^{5}$ The relative abundance of Bifidobacteriaceae was highest in exclusively breastfed infants and lowest in infants who were not breastfed. Infants at risk of being overweight had transient gut microbiota dysbiosis, including higher diversity and enrichment of Lachnospiraceae, during the first 100 days of life. Early microbial and metabolic alterations have also been associated with increased risk of childhood asthma in the CHILD cohort, ${ }^{6}$ and infant gut microbiota features were shown to predict food sensitization during the first year of life. ${ }^{7}$ These findings suggest breastfeeding may contribute to protection against these chronic diseases by modifying the gut microbiota, particularly during early infancy.

Breast milk contains many bioactive components that may influence health and development, the majority of which are not found in formula. Breast milk promotes the development of a healthy gut microbiota by providing probiotic bacteria and prebiotic nondigestible carbohydrates (i.e., human milk oligosaccharides [HMOs]) that select for beneficial bacteria. ${ }^{8} \mathrm{HMO}$ composition is highly variable between mothers. Data from the CHILD study indicate that genetic FUT-2 secretor status is a main determinant of HMO composition in breast milk, although other factors, including lactation stage, parity, season, and ethnicity, were also associated with variation in HMO concentrations. ${ }^{9}$ HMO diversity may be important for overall health outcomes. A study of 421 infants in the CHILD study found that the overall profile of HMOs in breast milk, rather than individual HMOs, was associated with food sensitization at 1 year of age. ${ }^{10}$ Current studies are analyzing breast milk samples from the CHILD cohort to measure other bioactive components, including fatty acids, endocrine hormones, and cytokines, which may play a role in obesity, allergies, and asthma.

In summary, data from the CHILD study, as well as previous research, indicate breastfeeding is associated with gut microbiota composition and reduced risk of chronic diseases, such as obesity and asthma. Multiple components of breast milk, including microbiota and HMOs, contribute to the beneficial effects of breastfeeding. The maternal determinants of milk composition and their impact on infant health are yet to be fully elucidated. Beyond milk composition and the microbiome, other factors related to breastfeeding, such as self-regulation and mother-infant bonding, may also affect health outcomes. These questions are the focus of ongoing and future research in the CHILD study.

\section{Acknowledgments}

The Canadian Institutes of Health Research (CIHR) and the Allergy, Genes and Environment Network of Centres of Excellence (AllerGen NCE) provide core support for the CHILD Study. M.B.A. is supported by the Canada Research Chairs program.

Analysis of breastfeeding and human milk in the CHILD Study has been supported by Research Manitoba, the Canadian Lung Association, the Canadian Respiratory Research Network, the Heart and Stroke Foundation, the Manitoba Children's Hospital Foundation, and the Manitoba Medical Services Foundation.

These entities have had no role in the design and conduct of the CHILD study; collection, management, analysis, and interpretation of data; or the preparation, review, or approval of the manuscript.

\section{Disclosure Statement}

No competing financial interests exist.

\section{References}

1. CHILD Study. 2019. www.childstudy.ca

2. Azad MB, Vehling L, Chan D, et al. Infant feeding and weight gain: Separating breast milk from breastfeeding and formula from food. Pediatrics 2018;142:pii:e20181092.

3. Azad MB, Vehling L, Lu Z, et al. Breastfeeding, maternal asthma and wheezing in the first year of life: A longitudinal birth cohort study. Eur Respir J 2017;49:pii:1602019.

4. Klopp A, Vehling L, Becker AB, et al. Modes of infant feeding and the risk of childhood asthma: A prospective birth cohort study. J Pediatr 2017;190:192-199.e2. 
5. Forbes JD, Azad MB, Vehling L, et al. Association of exposure to formula in the hospital and subsequent infant feeding practices with gut microbiota and risk of overweight in the first year of life. JAMA Pediatr 2018;172: e181161.

6. Arrieta MC, Stiemsma LT, Dimitriu PA, et al. Early infancy microbial and metabolic alterations affect risk of childhood asthma. Sci Transl Med 2015;7:307ra152.

7. Azad MD, Konya T, Guttman DS, et al. Infant gut microbiota and food sensitization: Associations in the first year of life. Clin Exp Allergy 2015;45:632-643.

8. Bode L. Human milk oligosaccharides: Every baby needs a sugar mama. Glycobiology 2012;22:1147-1162.

9. Azad MB, Robertson B, Atakora F, et al. Human milk oligosaccharide concentrations are associated with multiple fixed and modifiable maternal characteristics, envi- ronmental factors, and feeding practices. J Nutr 2018;148: 1733-1742.

10. Miliku K, Robertson B, Sharma AK, et al. Human milk oligosaccharide profiles and food sensitization among infants in the CHILD study. Allergy 2018;73:2070-2073.

Address correspondence to: Meghan B. Azad, PhD

Children's Hospital Research Institute of Manitoba Department of Pediatrics and Child Health University of Manitoba R3E 3P4 Winnipeg Canada

E-mail:meghan.azad@umanitoba.ca 